Prof. Dr. med. Herbert Kellner

Internist, Rheumatologe und Gastroenterologe,Schwerpunktpraxis für Rheumatologie und Gastroenterologie, München
Keywords:

Dyspnea, Aspirin-induced Asthma,

Osteoarthritis, NSAID, Coxibs

\title{
Wenn unter NSAR die Luft wegbleibt
}

Eine 64-jährige Frau behandelt eine schmerzhafte Kniegelenksarthrose selbst mit Ibuprofen. Bei einer orthopädischen Konsultation wird ihr empfohlen, die relativ wirkungslose Tagesdosis von 3 x 200 mg auf 1200-2400 mg zu erhöhen. Unter 1200 mg/d bemerkt die Patientin erstmals Atemnot und ein Beklemmungsgefühl. Trotzdem steigert sie in den folgenden Tagen die Dosis auf 1800 $\mathrm{mg} / \mathrm{d}$. Darunter kommt es zu einer akuten, einem Asthmaanfall ähnlichen Symptomatik, die einen Notarzteinsatz erforderlich macht. Bei der Anamnese berichtet die Frau, dass sie in der Jugend an Asthma bronchiale gelitten hat.

— Akut einsetzende Atemnot bei Einnahme von nicht steroidalen Antirheumatika (NSAR) weist in der Regel auf ein sogenanntes „Aspirin-Asthma“ hin. Mehr als 20\% der Erwachsenen und über 5\% der Kinder mit einem (intrinsic) Asthma entwickeln nach Einnahme von ASS innerhalb von Minuten bis Stunden eine akute Atemnot.

93-100\% der Patienten, die eine Intoleranz gegenüber ASS aufweisen, sind kreuzreaktiv gegenüber den traditionellen NSAR, insbesondere gegenüber Naproxen, Ibuprofen und Diclofenac, empfindlich (Tab. 1). Symptome können auch eine chronische (wässrige) Rhinitis, eine chronische Nasen- nebenhöhlenentzündung oder therapierefraktäre Nasenpolypen sein.

\section{Cox-1-Hemmung fördert Bronchokonstriktion}

Ursächlich für diesen „aspirinabhängigen" Krankheitskomplex soll die mit der ASS- oder NSAR-Einnahme verbundene unselektive Cox-1-Hemmung sein. Dadurch scheint das pulmonale Gleichgewicht zugunsten bronchokonstriktiver Mediatoren (Leukotriene) und zuungunsten bronchodilatierender Prostaglandine $\left(\mathrm{PGE}_{2}\right)$ verschoben $\mathrm{zu}$ werden. Im Verdachtsfall sollte in jedem Fall eine medikamentöse Reexposition vermieden werden. Durch gezielte Allergietestung (in anstei- gender Dosierung) kann die pulmonale Empfindlichkeit gegenüber ASS und NSAR ausgetestet werden.

\section{Therapeutische Alternativen}

Als therapeutische Alternative kommen bei degenerativen Gelenk- und Wirbelsäulenerkrankungen neben Paracetamol mit nur geringer „Kreuzreaktivität“ vor allem die analgetisch und antiphlogistisch wirksamen selektiven Cox-2-Hemmer infrage. Trotz entsprechenden (klassenpezifischen) Warnhinweises in der Packungsbeilage sind in kontrollierten Studien und Fallberichten bei weniger als 5\% der mit Coxiben behandelten Patienten mit „Aspirin-Asthma“ pulmonale Nebenwirkungen berichtet worden (Tab. 2).

Pulmonale Nebenwirkungen mit Atemnot werden bei der medikamentösen Rheumatherapie u.a. auch bei Methotrexateinnahme (interstitielle Pneumonitis) beobachtet.

Prof. Dr. med. Herbert Kellner Romanstr. 9, D-80639 München hk@prof-dr-kellner.de

\section{Tabelle 2}

Wahrscheinlichkeit der Kreuzreaktivität bei „Aspirin-Asthma“

$\begin{array}{lr}\text { Naproxen } & 100 \% \\ \text { Ibuprofen } & 98 \% \\ \text { Diclofenac } & 93 \% \\ \text { Paracetamol } & 7 \% \\ \text { Celecoxib } & <5 \%\end{array}$

Coxibe ohne Cox-1-Hemmung und ohne „Kreuzreaktivität"

Celecoxib

Etoricoxib

Ibuprofen

Naproxen

Diclofenac

NSAR mit Cox-1-Hemmung und Indometacin

Piroxicam 